\title{
What Do We Mean by the Meanings of Music?
}

\author{
PHILIP J. BARNARD [1] \\ MRC Cognition and Brain Sciences Unit, 15 Chaucer Road, Cambridge, UK, CB2 7EF
}

\begin{abstract}
Drawing upon a recent review of the topic by Cross and Tolbert (2009), this paper briefly illustrates the diversity of theories concerning the nature of meanings in music and the challenges that need to be resolved to advance the field. A scheme for layered macro- and micro-theories for neural, mental and behavioural systems is outlined to facilitate the development of a systematic and coherent body of theory. The core of the paper charts the evolutionary origins of a specific macrotheory of the organisation all the components of the human mind. This "mental architecture," known as Interacting Cognitive Subsystems (Barnard, 1985), incorporates not one, but two qualitatively distinct forms of meaning. Propositional meanings represent referentially specific ideas while implicational meanings encapsulate a yet more abstract and holistic form of meaning that blends conceptual material with the products of immediate distal and bodily sensations. While both forms of meaning interact in the interpretation and expression of musical meaning, such meanings are argued to be primarily implicational in nature. The paper concludes with a short discussion of how this approach might usefully be applied in the development of more precise specification of what music might mean in its various facets.
\end{abstract}

Submitted 2012 January 6; accepted 2012 July 13.

KEYWORDS: meaning, mental architecture, Interacting Cognitive Subsystems

IF you were informally to ask a sample of people in the street whether or not music meant something to them, it would be surprising if most did not rapidly answer in the affirmative. Music, after all, is pervasive in our modern culture and the cultures of our immediate forebears. Ask a sample of music experts or theoreticians and the answers would most probably neither be short nor uniform. The whole idea of the "meaning" of music is far from easy to pin down. As Cross and Tolbert (2009) note, issues that surround the nature of musical meaning have been central to the ways in which music has been explored and theorised. Yet, in spite of extensive debate there is little consensus.

\section{MULTIPLE DIMENSIONS OF MUSICAL MEANING}

In their perceptive review of proposals concerning meaning in music Cross and Tolbert (2009) draw our attention to numerous limitations of current positions and the challenges that the field must resolve. Meaning in music has multiple dimensions. Music definitely has "significance," and that significance can often only be grasped by considering the social context or culture in which a particular kind of music is enacted (Feld \& Fox, 1994). They also point out that music cannot so readily be seen to refer to an entity, property or relationship in quite the same way as language. Formal theories of semantics with a basis in logic (e.g. see Scruton, 1987) do not seem to be in position to provide a pertinent account of musical meaning. Equally, while variants of Pierce's classic semiotic distinctions between meanings rooted in iconic, indexical and symbolic relationships have also been applied to music (see Nattiez, 1991), Cross and Tolbert note that the status of music is ambiguous with respect to the categories at the heart of this framework. Yet other approaches to meaning propose that it is first and foremost grounded in bodily experiences (e.g. Johnson, 1987; Lakoff, 1987). This, in turn, provides a route to addressing specific phenomena such as entrainment. Nor has emotion been neglected. From classical times to the present, music can be shown to evoke affect (Juslin \& Sloboda, 2001). Musical meaning has even been linked to specific neural circuits also activated by linguistic meanings perhaps involving some common mediating factor such as imagery (Steinbeis \& Koelsch, 2008).

The challenges of resolving musical meaning, Cross and Tolbert argue, are unlikely to be met by one generally applicable theory or even by a single method. We are going to need new approaches within the psychological sciences that suggest ways of addressing meanings that are inherent or 
emergent in musical interactions and which also aim to account for meaning within an evolutionary framework (e.g. see Cross \& Woodruff, 2009). These challenges are by no means easy to address in a simple way - the significant number of attributes of musical meaning effectively call for a suite of theories that inter-relate not only diverse components of mind, but also show how those components of mind are constrained by human interactions with physical and animate entities as well as by the neural systems that underpin them.

\section{MEANING, SYSTEM LEVELS AND MACRO-THEORY}

Following Barnard, May, Duke \& Duce (2000), Figure 1 shows three levels at which pertinent systems can be analysed. These are neural architecture, mental architecture and behavioural architecture. Each level is an assembly (A) of basic units (B's) that themselves can each be decomposed into constituents (C). The basic units of mental architecture are processes, the basic unit of neural architecture electrochemical circuits and the basic units of behavioural architecture are animate agents and the physical entities they interact with in some setting - here they are musical instruments. Of course, defining the things that interact in a system is only a point of departure for formulating theories of how these systems actually behave - we also need to specify what the capabilities of the individual B's and C's are, what requirements need to be met for those capabilities to be used and the principles that govern the dynamic control and co-ordination of their changes of state - be those changes electrochemical, information states or a result of actions executed in a physical or social context.

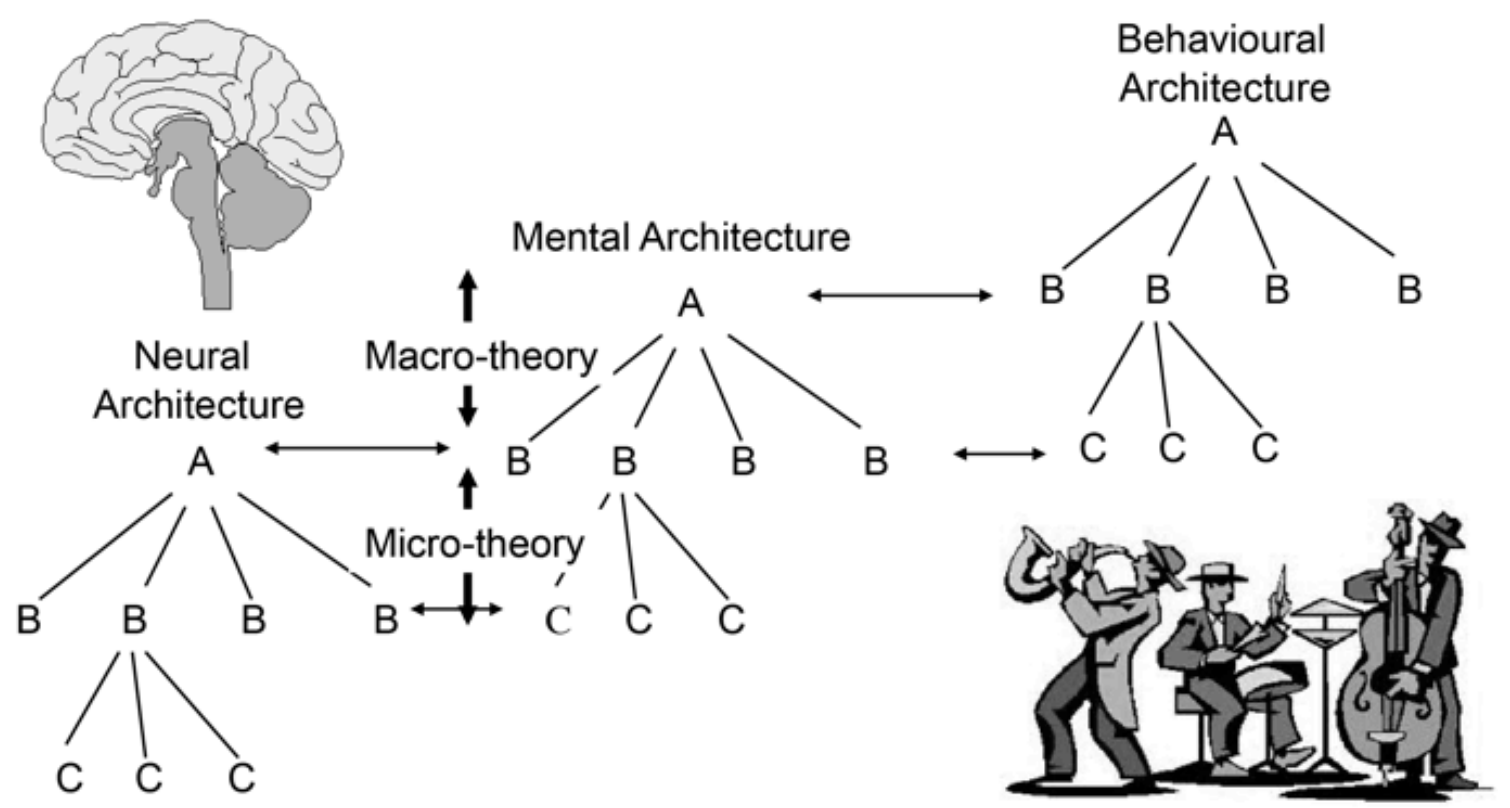

Fig. 1: Three system levels relevant to analyses of the nature of musical meanings.

While we are a long way from having theories of these systems that are either descriptively or explanatorily adequate, Figure 1 draws attention to two dimensions of theory formation. One dimension is captured by double-headed vertical arrows annotated "macro-theory" and "micro-theory." In the case of mental architecture micro-theories are theories of specific mental competences, or how the behaviour of the " $\mathrm{B}$ 's" is bottom-up constrained by properties of their " $\mathrm{C}$ 's." The " $\mathrm{B}$ 's" might be perception, language, attention, learning and memory or motor skills and there is often overlap in what these micro-theories address. Mainstream psychological science has focussed most of its effort on developing micro-theories at the expense of trying to formulate theories of how all of our mental competences work together. Furthermore, few theorists would agree on the exact categories of "B's" or "C's we should be using.

Many of the limitations of approaches to musical meaning summarised earlier can be traced back to the fact that the specific ideas tend to have been grounded in one or another micro-theoretic tradition. All of the different cognitive and affective attributes that have been debated in this context would seem to require resolution within macro-theory rather than by a collection of micro-theories that may be mutually irreconcilable. Horizontal doubled headed arrows, the second dimension of theory, indicate systematic relationship between analyses at different system levels. You can obviously have the same mental architecture embedded in very different behavioural architectures (or cultures). Variation in the capabilities and workings of mental architecture will be grounded in experience in a 
physical world of behavioural interactions and correlates of those experiences will propagate across all the basic components of mental architecture and the neural substrates on which they depend.

This paper will offer a way addressing issues associated with musical meaning from the specific perspective of a macro-theory of human mental architecture and how it evolved from a simpler form of mental architecture typical of most mammals. The analysis considers two qualitatively different types of meaning originally proposed by Barnard and Teasdale (1991) to analyse relationships between cognition, affect and embodiment. One form of meaning relates to the encoding of "propositions," whilst the other blends ideas, externally derived percepts and bodily experience to form "implicational meanings." These are more abstract schemata or models of deep multimodal interdependences that equate more with latent senses of meaning or knowing as well as feelings, intuitions and affect. Propositional and implicational meanings co-exist in mental architecture and depend one upon the other. While language skills are more clearly focussed on the form and expression of propositional meanings, musical expertise and aesthetic appreciation links most readily to implicational meanings.

To situate the arguments detailed below, music and language require both forms of meaning to interact. Musical meanings are best elaborated as the communication and appreciation of latent implicational meanings that are realised in interactions among multiple components of mental architecture, including propositional meanings. In relation to Figure 1, the actual realisations of these two types of meaning are in part constrained by influences from a specific culture (behavioural architecture), and in part by the evolutionary origins of our electrochemical circuitry (neural architecture).

\section{THE EVOLUTIONARY ORIGINS OF PROPOSITIONAL AND IMPLICATIONAL MEANINGS}

Many of the issues addressed in debates about musical meanings find direct parallels in analyses of psychopathologies where specific concepts and abstract feelings about self and others, emotions and embodiment all need to be factored into a comprehensive macro-theoretic account of mechanisms leading to, and sustaining, a particular condition such as depression. The distinction between propositional and implicational meanings has been most extensively elaborated in this context (Teasdale \& Barnard, 1993), while the evolutionary origins of these two forms of meaning and their processing has also been specified in some technical detail (Barnard, Duke, Byrne, \& Davidson, 2007; Barnard, 2010). Here, key aspects of the arguments will be summarised with an emphasis on those features most relevant to issues associated with inter-relating meaning and music.

Figure 2 diagrammatically depicts four subsystems (the B's of figure 1). This arrangement is proposed as the configuration of the minds of those mammals, such as wolves, that lack sophisticated cognitive capabilities (Barnard et al., 2007). There are three sensory subsystems (visual, body-state and acoustic) and a single multimodal subsystem highlighted in black. Information flows one way in this architecture from physical sensors through the sensory subsystems to the multimodal subsystem. This, in turn, controls what the effector systems of the body actually do. Naturally, each of these subsystems has constituent processes (the C's of Figure 1) detailed in other papers. Here, it is sufficient to note that this particular theory holds that each subsystem has its own memory and a dynamic "image" of the patterning of information over time, such as a tree branch waving in the wind. The information in that image can be attended to selectively and its content is the substance of what mammals can subjectively experience as "out there in the world" or "in the body" (sensory subsystems) or as more abstract senses or feelings (Multimodal subsystem). 


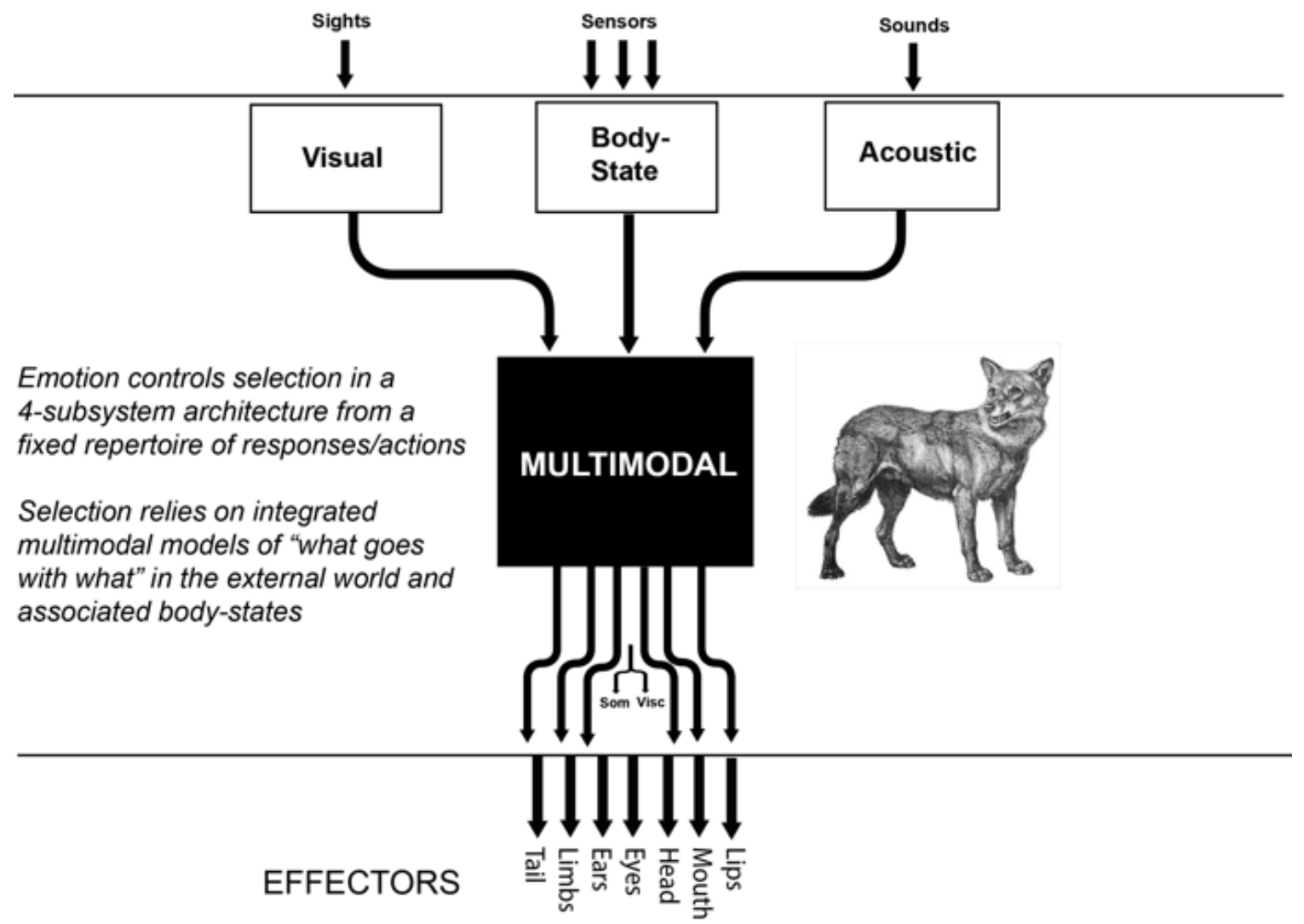

Fig. 2: A Four-subsystem mental architecture argued to hold for most mammals lacking any sophisticated cognitive capabilities. The abbreviations Som and Visc stand for Somatic and Visceral response systems in this and later figures.

On this view the individual subsystems each learn about what goes with what in the images they receive on the basis of rather simple statistical mechanisms - something like a principal components analysis with time as one of its dimensions (see Barnard et al., 2007 for details). So the sensory subsystems will effectively "do" perceptual learning for visual patterns, patterns of bodily sensation and auditory patterns while the multimodal subsystem will take the products of all those sensory analyses and "do" a higher order principal components analysis of deeper more abstract relationships of the kind that have been extensively studied in the paradigms of classical and instrumental conditioning. This tunes animal mental mechanisms to react adaptively in similar ways to things or events with similar properties and in different ways to things, events or circumstances that are different. Importantly, and in common with many other views proposing that emotion modules or programmes originally evolved to support the adaptive control of action (e.g. see Tooby \& Cosmides, 2000), affective markers of, for example, gratification, safety, or discomfort will guide selection of what to do from within a repertoire of fixed action patterns.

The multimodal subsystem of this architecture is building quite sophisticated "schemata" or statistical "models" of how to react in intricate context-dependent physical and social worlds whose precise characteristics can alter over time. These models must capture the essence of what goes with what and inherently assess the "significance" of current states along with when and how to react to them. A strong case can be made that the deep signature of this architecture has been preserved in an augmented form in our own more evolved mental architecture and that implicational meanings are rooted in the stuff of very basic multi-modal relationships that are carried through into the human appreciation of music. The fundamental properties of sights and sounds tell us a great deal about objects events and places (e.g. Jenkins 1985) as well as how to act in relation to them. Physics dictates that large or heavy objects, for example, will vibrate at low frequencies and smaller and lighter ones at higher frequencies. Many multimodal relationships of this type exist that animals and humans can make use of. 


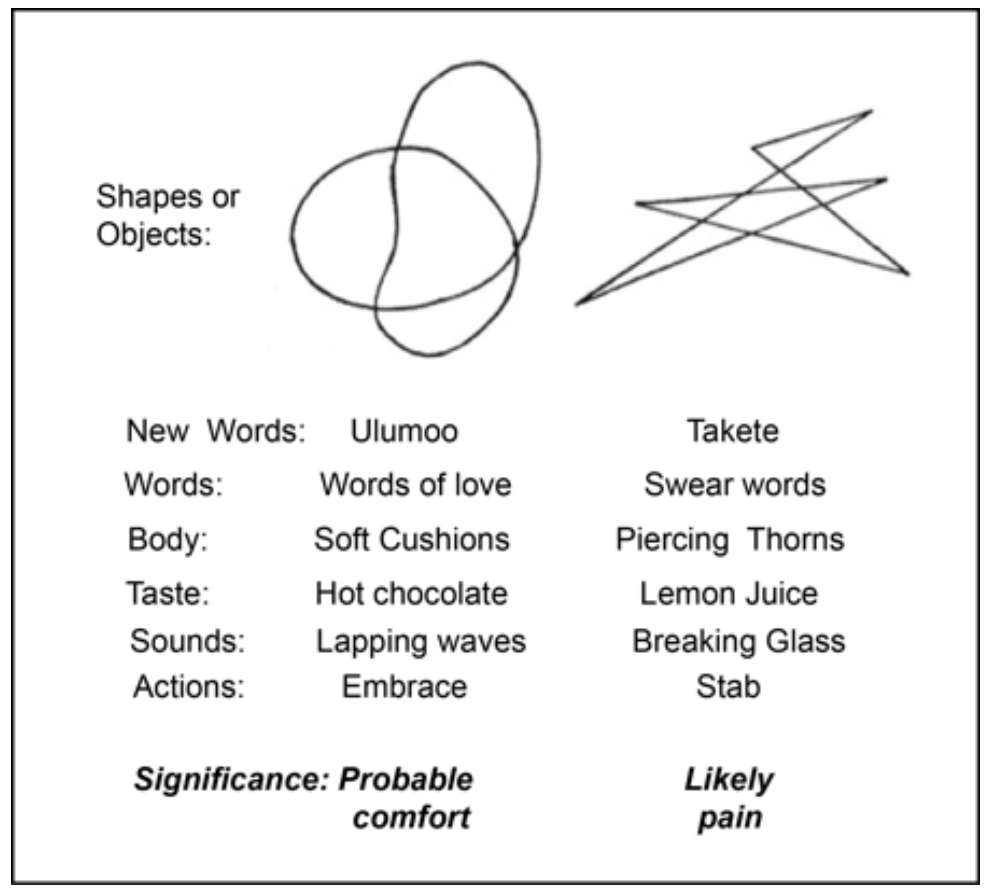

Fig 3: Multimodal patterns perhaps involving an abstraction inter-relating "acute" as opposed to soft or gentle transitions in vision, audition and body states with positive and negative affective markers.

It has been known for a long time that we humans capitalise on abstract properties of our multimodal experience. The upper part Figure 3 shows two graphics. Davis (1961) originally showed that young children from markedly different cultures across the world reliably associate the novel nonword sounds of Takete with the novel jagged shape and the sound of novel non-words like Ulumoo or Maluma with the equally novel more rounded and billowing shape. Similar regularities apply with known as opposed to novel words - expletives are quite likely to involve "hard" consonants while words of love tend to involve rather softer vowel sounds. More likely than not people would link Takete to the sound of breaking glass and Ulumoo to the more melodic sound of gentle waves lapping on a beach. It has recently been shown that the same considerations apply to associations between tastes and the sounds of words (Gallace, Boschin, \& Spence, 2011). Similar abstractions occur with movements involving purely abstract shapes (Heider \& Simmel, 1944). Abstract shapes moving in together in smooth and perhaps embracing patterns are interpreted as representing agents in contexts of social affiliation while those moving with abrupt stabbing movements are interpreted as behaviours more typical of antagonist social contexts (Tavares, Lawrence and Barnard, 2008). Jagged objects like shards of glass or thorns can pierce the skin and cause discomfort while stroking fur or being enveloped in soft billowing cushions is more likely to be a source of comfort. These are deep configural properties that enter into dendritic relationships rather than superficial properties of cleanly delineated scope. At best, we can over-simplify the essence of such patterns as involving families of "acute transitiondiscomfort" relationships or families of "smooth transition-comfort" relationships.

We experience such cognitive-affective patterns as systematic 'senses or feelings' that yield reliable and measurable behaviours yet are extraordinarily difficult to realise as specific propositional meanings (Teasdale \& Barnard, 1993). By extension we use linguistic phrases like "he has a sharp tongue" or "that is well-rounded argument" to convey intricate, and in this case, evaluative meanings. However, while the dimensions of deep schemata can be richly expressed in language through metaphor and other rhetorical devices, the actual multimodal regularities that give rise to them may encompass many dimensions that can only be captured through some high dimensional statistical analysis such as latent semantic analysis (e.g. Landauer \& Dumais, 1997).

It would seem reasonable to argue that abstraction and modelling of significant aspects of the "acute-transition-discomfort" patterns and "smooth transition-comfort" patterns across sights, sounds and bodily sensations would be well within the modelling capabilities of the multimodal component of the four-subsystem architecture shown in Figure 2. Similarly, the sounds of slow irregular footfall of a small deer, coupled with the sight of asymmetric biological motion, could come to be yoked as signifying a more rather than less vulnerable prey, perhaps with a weakness or injury, and hence a good prospect to target in a hunt. The examples in Figure 3 that rely on non-words, expletives and the interpretation of abstract shapes "behaving" in cartoon movies would be unlikely to apply in foursubsystem wolf cognition, since the architecture of its mind lacks basic units to support the processing of more advanced forms of spatial, linguistic and semantic imagery. 
The core argument proposed by Barnard et al. (2007) is that spatial, linguistic and semantic imagery and their allied processes emerged out of an evolutionary trajectory in which five new subsystems were added to the configuration shown in Figure 2. At each stage, in a pattern not unlike cell division in biology, the original Multimodal subsystem spawns a new daughter subsystem. Each addition results in a new configuration that brings a package of mental and behavioural capabilities and each addition changes the content of, and differentiation within, the multimodal syntheses that underpin cognition and affect. Here key features of that trajectory will be summarised, the details of semantic and affective differentiation and the emergence of intricate attentional and "executive" processes have been fully documented elsewhere (Barnard, 2010; Barnard et al., 2007; Byrne et al., 2004).

Figure 4 shows an architecture in which the core components of our original four-subsystem architecture are augmented by four new ones and the order of addition is indexed by the white number sequence at the lower right of each of the boxes filled in grey. The principles proposed for subsystem differentiation entail a single trajectory of architectural evolution from our last common ancestor with extant great apes. When following the ensuing text, it is important to note that descriptions of, for example, a six-subsystem architecture effectively invite readers to imagine a variant of this Figure in which subsystems seven and eight are absent.

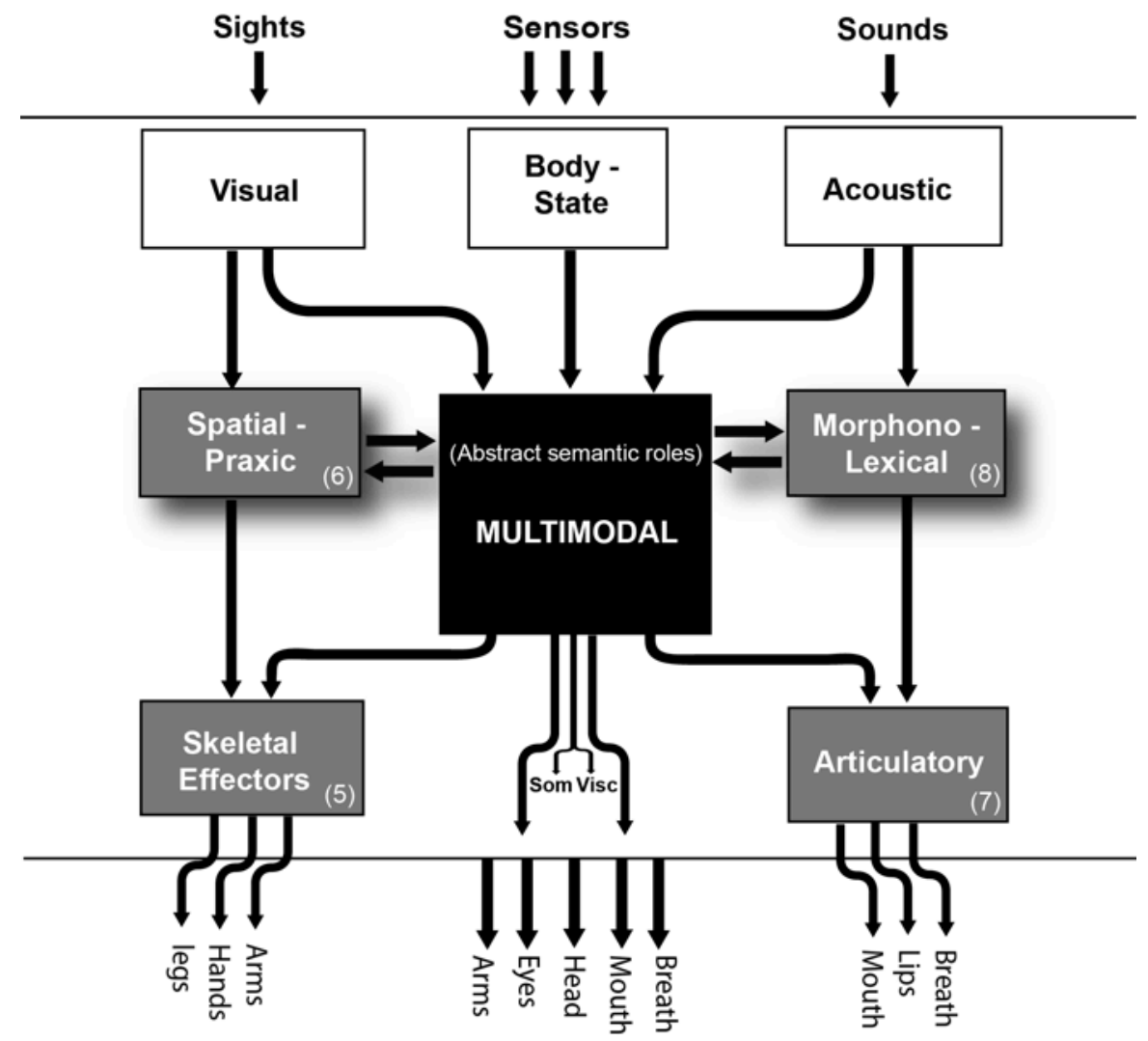

Fig 4: An eight-subsystem architecture for the immediate hominin ancestors of modern humans (simplified from Barnard et al., 2007).

The first segment of the overall trajectory involves the development of more advanced skills in "spatial-praxis" and its internal representation. First, some precursor species of monkey is presumed to have evolved intricate manual dexterity and the detailed control of finger movement in the context of wider skeletal musculatures - such re-configurations of balance required when reaching to twist off a fruit. The product of this differentiation would have been a new subsystem specialised for the control of skeletal effectors (Figure 4, box indexed 5).

Importantly, once in place, the temporal patterning of skeletal re-configurations controlled by this effector subsystem would be sensed within the body-state subsystem and their first order patternings, or derivatives, sent on to the Multimodal subsystem. In parallel, of course, the monkey would be "seeing" the action and the first order derivatives of the dynamic changes in visual patterning would also be sent to the Multimodal subsystem. Here the underlying neural networks would be able to abstract properties of the changes that were shared by both vision and body state dynamics - abstract invariants relating to, for example, twisting, inverting, timing, size reduction and so on. These abstractions would initially form the seeds of a "spatial-praxic" coding system. Once a fully 
differentiated coding system stabilises, a new daughter subsystem would emerge (Figure 4, box indexed 6). The technical details of the differentiation are given in Barnard et al. (2007). Of note here is the fact that the new sixth subsystem inter-mediates between vision, effector control and multimodal synthesis. It represents a gateway for the interpretation of visual patterns over time as well as their implementation as actions in the world. Spatial-praxic encodings represent that part of the original multimodal subsystem concerned with something akin to deeper "structural descriptions" of changes in visual states and how to realise them in muscular control. Three critical new capabilities underpinning the behaviour of mental architecture emerge at this point.

First, there is a new level of abstraction - the multimodal subsystem now receives information that has undergone two preceding types of analysis. The first order sensory analysis is followed by a more abstract spatial-praxic analysis before being passed to the multimodal subsystem. Whereas the four-subsystem architecture had just two levels of analysis the multimodal component of a sixsubsystem architecture can now compute a third level of abstraction that relates actions in physical space to other multimodal elements.

Second, the four-subsystem architecture involved what amounts to one-way traffic from sensation to action control. The six-subsystem architecture now supports an internal cycle - a reciprocal mapping between the processing of multimodal images and spatial-praxic ones. This mental architecture has a form of visual imagery that can occur concurrently with the control of overt action and supports "re-ordering" of action elements. This architecture can "think" spatially while doing. Third, the now deeper derivatives of abstract structural descriptions of actions in space (i.e. movement phrases) can be linked with affect in the multimodal subsystem rather than just similarities in movement based on first order sensations. This architecture can in principle have a feeling about gestures that share a common abstract composition.

These three properties, Barnard et al., (2007) argue, are sufficient to account for the more advanced capabilities of modern great apes - including programme level imitation of food processing, tool use, advanced working memory for visual sequences, mirror image recognition of "self," some theory of mind for the actions of others, and the cultural transmission of certain forms of almost dancelike and non-food related actions carried out in affectively marked contexts. All of these are arguably grounded in purely spatial-praxic skills and were all in place in the minds of our last common ancestor with extant great apes.

Great apes are known to have a restricted range of vocalisation, as would our last common ancestor. The case advanced by Barnard et al. (2007) is that the process simply repeated in the auditory-vocal domain for other hominins from whom we are descended. First, as with manual dexterity, differentiation of vocal utterances spawned an articulatory subsystem (Figure 4, box indexed 7) for the intricate and co-ordinated control of multiple muscular components: vocal cords, lips, tongue, timing of breath etc. Once in place the internal bodily feedback of changes allied to vocalisation can be inter-related within the Multimodal subsystem with what is heard, not only in "speech by self," but with speech and related sounds generated by others. Just as with the emergence of a spatial-praxic coding system, the invariants underlying vocal articulation and heard sound lead to the emergence of a "morphonolexical" subsystem (Figure 4, box indexed 8). This captures abstract structural descriptions of utterances, allows mental imagery of sounds and voices "in the head," decomposition and reordering of the elements of vocal phrases and the assignment of emotion to abstract properties of what is said as well as what is seen. This architecture has a form of productive linguistic communication.

The full eight-subsystem architecture of Figure 4 can do a lot more at one and the same time than the four-subsystem architecture of Figure 2 could do. In principle, it could give really quite complex verbal "instructions" while visually demonstrating the actions required in manufacturing a spear or stone tool. In this architecture, both vocal and skeletal actions are centrally co-ordinated from a single multimodal subsystem. Actions of others could be vocally rewarded or discouraged. The deepest abstraction within the Multimodal subsystem remains at three levels. However, the third level now inter-relates abstract structural descriptions of re-orderable visual dynamics and verbal inputs with their affect and cognitive significances. This is a key precondition for the final emergence of truly abstract semantic roles.

As with each of the prior stages along this hypothetical evolutionary trajectory, the multimodal subsystem operates by seeking to establish and make use of invariant patterning underlying the inputs it receives. With the four subsystem architecture these would be confined to the here and now of sensory properties relevant for selecting among fixed action patterns. With the six-subsystem architecture, the demands of, for example, tool manufacture would require distinguishing physical properties of cores and hammer stones. Action selections guided by multimodal distinctions would have to be made in the partitioning of meat or fruit by slicing with a stone flake or partitioning kernels from nutshells by pounding a hammer onto a nut located on an anvil and so on. Byrne et al. (2004) note that distinctions among the generic semantic roles proposed in case grammars (e.g. Fillmore, 1968) such as agent, object, or instrument roles, as well as other semantic properties, are implicit in the 
behaviours of chimpanzees but their presence does not mean we have to propose that they really rely on "semantic" abstractions.

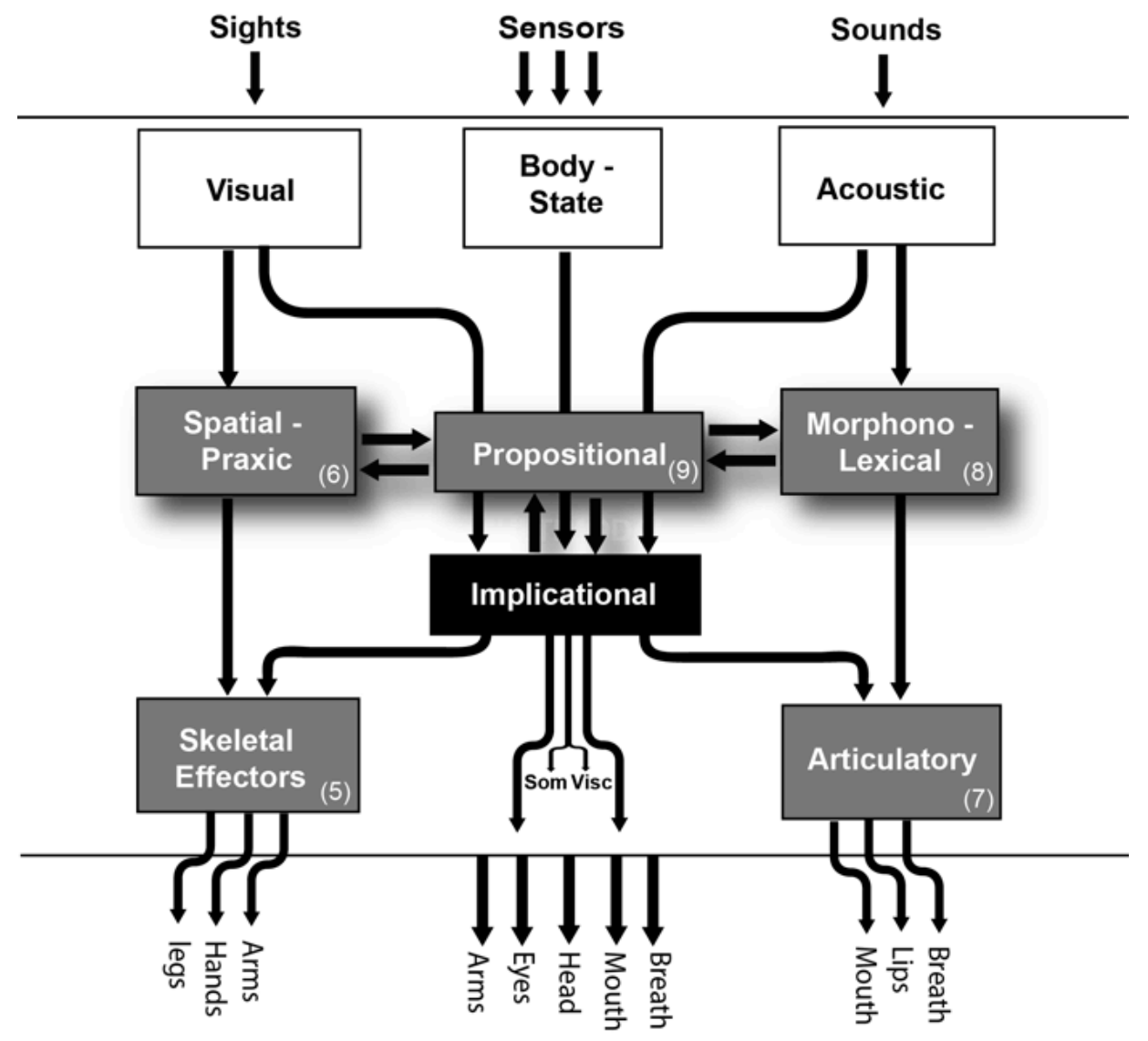

Fig. 5: Propositional and implicational meaning within the nine-subsystem architecture proposed for our own mental architecture (simplified from Barnard et al., 2007).

The eight-subsystem architecture is the crucible in which a truly abstract semantic coding system can be forged. Here the inputs to the Multimodal subsystem now include the products of analysing invariant structures in spatial-praxic dynamics and invariants underlying phonologically expressed phrases. Further, the referent of arbitrary phonological forms can only be grasped by intersecting invariant patterns that have that have systematically co-occurred within the Multimodal subsystem. The product is a semantic coding system for propositional meanings. Something like the case grammar distinctions implicit in chimpanzee behaviour come to form a stable coding system and enable a final, ninth daughter subsystem to emerge that explicitly represents propositional meanings (Figure 5).

The emergence of the ninth subsystem brings with it an enormous step change in cognitive capability. As with the emergence of the sixth subsystem, the transition to nine subsystems endows the architecture with the capability to abstract deeper regularities. These can now be four layers rather than three layers "deep." The longest chain of processing now goes though sensory subsystems $\left(1^{\text {st }}\right.$ order abstraction), through a layer of structural description ( $2^{\text {nd }}$ order abstraction), followed by propositional analysis ( $3^{\text {rd }}$ order abstraction), and into a final multimodal synthesis of $4^{\text {th }}$ order abstract patterns that underlie recurrent patterns in propositional meanings that are blended with the products of sensory processing (Vision, Body-states and Audition). At the outset it was pointed out that the analysis would show that the minds of modern humans retained the signature of the four-subsystem architecture of Figure 2. To mark this continuity, the implicational subsystem of Figure 5 retains the black shading of all its evolutionary precursors and the original flows from the three sensory subsystems. As with all previous transitions affect is retained in its original place - except now the markers of affect can attach not just abstractions of external states but to abstract ideas about the self, the world and others.

The owner of this architecture can feel success and failure in addition to the more basic emotions. In this scheme propositional meanings lack affective charge and the wider system can support multiple meanings. As Teasdale and Barnard (1993) point out, the depressed owner of a ninesubsystem architecture can "feel in their hearts" all of the implicational markers of "self-as-failure" whilst propositionally knowing "in their head" that they have attributes of success that are indisputably 
"true." The implicational meaning system of the nine-subsystem architecture supplements information directly derived from the senses with conceptually derived information. It has not only "feelings" about intricate states, the content of those implicational mental states are the stuff of abstractions underlying knowledge - intuitions, wisdom and deep expertise in, for example, science, chess, literature and, of course, art, religion, dance or music.

The emergence of the ninth subsystem also brings the ability for the human mind to do more things at one and the same time. The interaction between propositional and implicational meanings has been referred to as the "central engine of human ideation" (Teasdale \& Barnard, 1993). This enables the owner of this kind of architecture to think about ideas whilst walking, talking and chewing gum. The basic units are configured in a way that enables a modern human to think about how to make a better tool, whilst physically making the tool and humming a song at the same time. Within the dialogue between propositional and implicational meanings, the elements of 'ideas' can routinely be formed, re-composed and re-organised into either recurrent patterns known to be useful or into novel innovative forms that can be evaluated in mental simulation or by testing them in a real-world context of actual rather than imagined behavioural architectures.

A seven-subsystem architecture was most likely in place for one or more species of Homo during the era of $H$. erectus, while later archaic species such as $H$. heidelbergensis and $H$. neanderthalensis are argued to have possessed eight-subsystem minds. Only H. sapiens sapiens is regarded as having developed the full complement of nine-subsystems with two qualitatively distinct types of meaning.

\section{MACRO-THEORIES, MUSIC AND MEANINGS}

The nine-subsystem architecture thus defines the components of a candidate macro-theory for the modern human mind (the B's of Figure 2) and how they are configured as an architectural assembly (A). The allied definitions of the coding systems constrain how we formulate more specific claims about our mental capabilities and potential. It is a candidate theory that has seen applications in a number of areas within basic and applied theory (e.g. see Barnard, 1999; Barnard et al., 2000; Barnard, 2004; Barnard \& Bowman, 2003). Its particular form has been validated in only a limited range of experimental tests, and it remains a long way from being a fully mature "explanatorily adequate" theory. The fact that it has been applied across the domains of attention, language, memory, as well as meanings in cognition and emotion nonetheless provides some confidence of its potential descriptive range. Conceptually, it is also important to note that re-configurations of mental architecture bring a package of emergent properties rather than isolated features that engender specific evolutionary advantages. In the specific context of debates concerning the fundamental properties of musical meaning, the layered framework of systems analysis shown in Figure 1, at least enables us to address some of the key challenges laid out by Cross and Tolbert (2009).

One challenge specifically related to a need for multiple theoretical perspectives and methods for addressing meaning. The analysis of mental architecture suggested here makes claims about how meaning is encoded and abstracted from sensory experience. A macro-theory of mental architecture constrains but does not determine what is and isn't meaningful in a specific culture. Hence the layered framework of systems analysis of Figure 1 assigns the problem of specifying cultural meanings to macro-theory and micro-theory in behavioural architectures rather than mental ones - along with their methods for analysing social and physical interactions in real-world contexts. What a macro-theory of mental architecture should help us to achieve is systematic development work on the diverse and rather dendritic aspects of the problem of specifying cognitive and affective attributes of musical meanings.

All of the components of the nine-subsystem architecture would be recruited in one way or another when listening to music, making music, just talking about it or even teaching it. Musical performance, be it instrumental or vocal, relies on highly practiced expertise in either articulatory or skeletal effector subsystems as well as the feedback of visual auditory, and body state components to the multimodal subsystems. Actions must be accurately selected and titrated, sequenced, timed with suprasegmental features being generated and monitored. The specific macro-theory proposed here, and provided with the kind of evolutionary justification requested by Cross and Tolbert (2009), has quite specific and potentially testable, ramifications for our understanding of musical meaning.

The opening remarks drew attention to the idea that musical meanings most readily related to implicational rather than propositional meanings. The evolutionarily grounded argument now enables more precision to be given to the idea that meanings in music are best seen as the communication and appreciation of the contents of latent implicational schemata that are realised in interactions with multiple components of mental architecture, including propositional meanings.

As should be clear from their evolutionary origins, the nature and origins of implicational representations directly confront the issue of why music is significant, linked both to affect and direct perception of physical states of the world and body, while remaining "hard to pin down" conceptually. The original elaboration of the concept of implicational meanings, as applied to depressive 
psychopathology and how it differs from propositional meanings (Teasdale \& Barnard, 1993), closely mirror the aspects of debates about musical meanings. Teasdale and Barnard note that forms like poetry, metaphor and parable more accurately capture the rich yet latent high dimensionality of implicational meanings. As one thought experiment they took some well-known poetry and translated it into an alternative "propositional form" matched phrase by phrase.

So the lines from La Belle Dame Sans Merci (Keats):

"Oh what can ail thee knight at arms,

Alone and palely loitering?

The sedge has wither'd from the lake, And no birds sing",

becomes the prose:

What is the matter armed old-fashioned soldier, standing by yourself and doing nothing with a pallid expression? The reed-like plants have decomposed by the lake and there are not any birds singing.

While the actual individual phrases map reasonably well one on to another, the poetry conveys a sense of haunting loneliness not shared by the "prose" translation. The two texts express the same propositional meanings but different implicational schemata.

Of course poetry, metaphor and parable largely depend on the subsystems that deal with spoken or written language. But the architecture of Figure 5 provides means of distinguishing alternative routes to meaning. Eight of the nine subsystems in this Figure are no more than one processing stage away from direct sensation or effector control. Mental processing within these subsystems is inherently linked to first order abstractions of stuff that behaves either out there in the world or within the body itself. The exception is the Propositional subsystem. This form of meaning receives no inputs that are necessarily constrained by the first order derivatives of stuff co-present out there in the world or in the body. It communicates only with other central subsystems and controls the form and content of imagery in the mind - very much like the central executive in Baddeley's classic model of working memory acts to controls a phonological loop and visuo-spatial scratch pad across a range of disembodied cognitive "tasks" (see Baddeley, 2007).

Whilst we can talk "about" musical meaning in propositions or recruit abstract structural description of vocal or spatial-praxic phrases, the experience of music is thus arguably more closely allied to implicational schemata. Such implicational schemata encapsulate "embodied" dimensions. They also directly control visceral, somatic and other globally patterned responses (see Figure 5). As noted previously such schemata are directly constrained by distal visual and auditory patterns that optionally link ideas to affect. When synthesised with the products of propositional meanings implicational schemata can encapsulate abstract model of self, the world and others.

This form of synthesis thus also provides a basis for addressing known empirical phenomena and making new predictions. In particular, it provides a clear basis for explaining phenomena such as entrainment or social contagion (e.g. see Clayton, Sager \& Will, 2004) as well as the latent effects of background music on purchasing behaviour in supermarkets (e.g. see Milliman, 1982). Although much music is familiar, the interaction between implicational schemata and propositional meanings supports innovation. This central engine of ideation could be argued to provide a route for developing more precise ideas about the basis of musical creativity or evaluative criticism.

In the broader context of the nine-subsystem architecture, theories of both language and music would necessitate at least some reference to both forms of meaning. However, the analysis of language behaviours is inherently linked to the communication of propositional content. It is used when referentially specific attributes need to be pinpointed to guide one's own behaviour or the behaviour of others. In contrast, key aspects of music, dance and art may function not so much to pinpoint referentially specific meanings but to draw participants into a region of particular senses of meaning or to feel affect and knowing about some broad region of "deep stuff." Such senses of meaning may serve a whole variety of personal and social functions. Importantly, propositional meaning emerged to support the sharing specific meanings in a manner that can be devoid of affect. Implicational meanings, on the other hand, are inherently generic. Since they are felt senses of an individual's existential state in a real world context and with a particular body state in place, such senses of meaning are inherently situated and "subjective." Indeed, the deep meanings of music may resist detailed description and sharing precisely because this property holds.

The kind of formulation outlined here is self-evidently far short of a fully-fledged theory. It is a sketch that would require multiple cognitive and affective "properties" of the type discussed in the context of Figure 3 (Takete \& Ulumoo/ affiliation and antagonism) to be tested and worked out with 
detailed analyses of a range of musical forms. The expert knowledge required is beyond that possessed by the current author. However, in the wider spirit of interdisciplinary dialogue, it is hoped that the very ideas of roles for well-specified macro-theory and two qualitatively distinct forms of meaning can provoke further productive debate concerning the meanings of music.

\section{END NOTES}

[1] The theoretical work described here was supported by the UK Medical Research Council under project code MC_US_A060_0022. The work has also benefitted substantially from an enduring sci-art collaboration with Scott deLahunta, director of R-Research at Wayne McGregor | Random Dance, Sadler's Wells, London.

\section{REFERENCES}

Baddley, A. (2007). Working memory, thought, and action. Oxford: Oxford University Press.

Barnard P.J. (1985). Interacting cognitive subsystems: a psycholinguistic approach to short-term memory. In A. Ellis (Ed.), Progress in the psychology of language, vol. 2. London: Erlbaum, pp. 197258.

Barnard, P.J. (1999). Interacting cognitive subsystems: modeling working memory phenomena within a multi-processor architecture. In A. Miyake \& P. Shah (Eds.), Models of working memory. Cambridge: Cambridge University Press, pp. 298-339.

Barnard, P.J. (2004). Bridging between basic theory and clinical practice. Behaviour Research and Therapy, Vol. 42, pp. 977-1000.

Barnard, P.J. (2010). From Executive Mechanisms Underlying Perception and Action to the Parallel Processing of Meaning. Current Anthropology, Vol. 51, S1, pp. S39-S54.

Barnard, P.J., \& Bowman, H. (2003). Rendering information processing models of cognition and affect computationally explicit: Distributed executive control and the deployment of attention. Cognitive Science Quarterly, Vol. 3, No. 3, pp. 297-328.

Barnard, P.J., \& Teasdale, J.D. (1991). Interacting cognitive subsystems: A systemic approach to cognitive-affective interaction and change. Cognition and Emotion, Vol. 5, pp. 1-39.

Barnard, P.J., May, J., Duke, D., \& Duce, D. (2000). Systems, interactions and macrotheory. ACM Transactions on Human-Computer Interaction, Vol. 7, No. 2, pp. 222-262.

Barnard, P.J., Duke, D.J., Byrne, R.W. \& Davidson, (2007). Differentiation in cognitive and emotional meanings: an evolutionary analysis. Cognition and Emotion, Vol. 21, No. 6, pp. 1155-1183.

Byrne, R.W., Barnard, P.J., Davidson, I., Janik, V.M., McGrew, W., Miklosi, A., et al. (2004). Understanding culture across species. Trends in Cognitive Sciences, Vol. 8, pp. 341-346.

Clayton, M., Sager, R., \& Will, U. (2004). "In time with the music: The concept of entrainment and its significance for ethnomusicology". ESEM Counterpoint 1, pp.1-82.

Cross, I. \& Tolbert, E. (2009). Music and meaning. In S. Hallam, I. Cross \& M. Thaut (Eds.) The Oxford Handbook of Music Psychology. Oxford, Oxford University Press, pp. 24-34.

Cross, I. \& Woodruff, G.E. (2009). Music as a communicative medium. In R. Botha \& C. Knight (Eds.) The prehistory of language. Oxford: Oxford University Press, pp. 113-144.

Davis, R. (1961) The fitness of names to drawings. British Journal of Psychology, Vol. 52, pp. 259268.

Feld, S., \& Fox, A.A. (1994). Music and language. Annual Review of Anthropology, Vol. 23, pp. 25-53. 
Fillmore, C. (1968). The case for case. In E. Bach \& R. Harms (Eds.), Universals in linguistic theory. New York: Holt, Rinehart, \& Winston, pp. 1-88.

Frijda, N.H. (2004). Emotions and action. In A. S. R. Manstead, N. Frijda, \& A. Fischer (Eds.), Feelings and emotions: The Amsterdam symposium. Cambridge, UK: Cambridge University Press, pp. 158-173.

Gallace, A., Boschin, E. \& Spence, C. (2011). On the taste of "Bouba" and "Kiki": An exploration of word-food associations in neurologically normal participants. Cognitive Neuroscience, Vol. 2, No.1, pp. 34-46.

Heider F, Simmel M. 1944. An experimental study of apparent behaviour. American Journal of Psychology. Vol. 57, pp. 243--259.

Jenkins, J.J. (1985). Acoustic information for objects, places, and events. In Warren, W. H., \& Shaw, R. E., (Eds.), Persistence and change. Proceedings of the first international conference on event perception. Hillsdale, NJ: Erlbaum, pp. 115-138.

Johnson, M. (1987). The Body in the Mind: The Bodily Basis of Meaning, Imagination and Reason. Chicago: Chicago University Press.

Juslin, P., \& Sloboda, J.A. (Eds.). (2001). Music \& emotion: theory and research. Oxford: Oxford University Press.

Lakoff, G. (1987). Women, fire and dangerous things. Chicago: University of Chicago Press.

Landauer, T.K., \& Dumais, S.T. (1997). A solution to Plato's problem: The latent semantic analysis theory of the acquisition, induction and representation of knowledge. Psychological Review, Vol. 104, pp. 211-240.

Milliman, R.E. (1982) Using background music to affect the behavior of supermarket shoppers. The Journal of Marketing, Vol. 46, pp. 86-91.

Nattiez, J.-J. (1990). Music and discourse: toward a semiology of music. Princeton, NJ: Princeton University Press.

Scruton, R. (1987). Analytical philosophy and the meaning of music. Journal of Aesthetics and Art Criticism, Vol. 46, pp. 169-176.

Steinbeis, N., \& Koelsch, S. (2008). Shared Neural Resources between Music and Language Indicate Semantic Processing of Musical Tension-Resolution Patterns. Cerebral Cortex, Vol. 18, pp. 11691178.

Tavares, P., Lawrence, A.D., and Barnard, P.J. (2008). Paying Attention to Social Meaning: an fMRI Study. Cerebral Cortex, Vol. 18, No. 8, pp. 1876-1885.

Teasdale J.D. \& Barnard, P.J. (1993). Affect, cognition and change: remodeling depressive thought. Hove: Erlbaum.

Tooby, J. \& Cosmides, L. (2000). Evolutionary psychology and the emotions. In Handbook of emotions. 2nd edition. M. Lewis and J. Haviland-Jones, Eds. New York: Guilford, pp. 91-115. 